\title{
Resilienz - Geschichte, Modelle und Anwendung
}

\author{
Leonhard Thun-Hohenstein · Kerstin Lampert • \\ Ulrike Altendorfer-Kling
}

Online publiziert: 29. Januar 2020

(C) Der/die Autor(en) 2020

Zusammenfassung In diesem Beitrag der Zeitschrift für Psychodrama und Soziometrie wird der Begriff der Resilienz beschrieben, eine zusammenfassende Definition gegeben und seine Anwendung dargestellt. Es werden die Resilienzfaktoren und verschiedene Modelle mit dem Versuch, eine vereinheitlichte Zusammenschau zu geben, beschrieben. Auf die Nutzung des Resilienzbegriffes im Psychodrama wird ebenfalls eingegangen. Zuletzt wird auch eine kritische Sichtweise auf die Anwendung des Modells in die Diskussion einbezogen.

Schlüsselwörter Psychodrama $\cdot$ Resilienz $\cdot$ Biopsychosoziales Modell · Widerstandsfähigkeit · Salutogenese

\section{Resilience—history, models and application}

\begin{abstract}
This article for Zeitschrift für Psychodrama und Soziometrie describes resilience, gives definitions and interpretations as well as models of resilience. This article provides an overview over criteria, factors and models of resilience, trying to give an integrated view of resilience. Also, the use of resilience in psychodramatic theory and practice is discussed. The use of the concept of resilience in psychodrama therapy is presented. Finally, critical developments of the use of the topic of resilience are discussed.
\end{abstract}

Keywords Psychodrama $\cdot$ Resilience $\cdot$ Biopsychosocial model $\cdot$ Resistance Salutogenesis

Prim. Univ. Prof. Dr. L. Thun-Hohenstein $(\bowtie)$

Universitätsklinik für Kinder- und Jugendpsychiatrie, Paracelsus Medizinische Privatuniversität,

Christian Dopplerklinik, Ignaz Harrerstr. 79, 5020 Salzburg, Österreich

E-Mail: L.Thun-Hohenstein@salk.at 


\section{Geschichte des Begriffs Resilienz}

Der Begriff Resilienz leitet sich vom lateinischen resilire (für zurückspringen oder abprallen) ab. Ursprünglich war damit die physikalische Fähigkeit eines Körpers gemeint, nach Veränderung der Form wieder in seine Ursprungsform zurückzuspringen. Resilienz wird ebenfalls in verschiedenen gesellschaftlichen Bereichen verwendet, beispielsweise im Ingenieurwesen, der Energiewirtschaft, als Resilienz von Ökosystemen und eben auch in der Psychologie.

Die Ersterwähnung des Begriffes Resilienz wird dem Psychologen Jack Block (1977) zugeschrieben. Seine Arbeit beschäftigte sich mit Kindern im Kleinkindalter, bei denen er die Ego-Kontrolle und die Ego-Resilienz analysierte und sie im Alter von sieben Jahren nachuntersuchte. Hoch-Ego-Resiliente Kinder wurden von ihren LehrerInnen und KindergärtnerInnen als empathischer, fähiger mit Stress umzugehen, intelligenter und emotional situationsangepasster, als sich selbst akzeptierend, Neuigkeiten suchend, kompetent, kreativ und weniger ängstlich beschrieben. Diese Kinder waren auch weniger in Konflikte verwickelt, misstrauten weniger und benötigten seltener Rückversicherungen.

Die amerikanische Forscherin Emmy Werner hat wohl am grundlegendsten zur Resilienzforschung und Einführung des Begriffs in die Psychologie beigetragen. Sie untersuchte eine gesamte Geburtskohorte der Insel Kauai und verfolgte diese Gruppe jahrzehntelang. In ihrer 1989 erschienen Arbeit (Werner 1989) beschreibt sie eine Gruppe von Kindern, die es im Vergleich zu anderen Kindern trotz ähnlich belastender Faktoren geschafft hatten, ein zufriedenstellendes, erfolgreiches und gesundes Leben zu führen. Werners Studien ermöglichten Entwicklungsprozesse neu zu sehen und lieferten neue Erkenntnisse über die Entwicklung im Sinne eines dynamischen Prozesses. Andere Studien dieser Zeit, wie die von Norman Garmezy (1991) und Ann Masten (Garmezy und Masten 1984) über die Kinder von schizophrenen PatientInnen, ergaben ebenfalls Hinweise auf entsprechende Resilienzfaktoren. So konnten sie zeigen, dass Resilienz nicht nur eine Frage der Ausstattung ist, sondern $\mathrm{zu}$ verschiedenen Zeitpunkten durch diverse Faktoren wirksam wird.

Parallel zu Werners Kauai-Studie untersuchte Anton Antonovsky (1997) den psychischen und körperlichen Gesundheitszustand von Frauen, die das Konzentrationslager überlebt hatten. Er fand knapp 30\% der Frauen aus den KZs, die trotz dieser Erfahrungen gesund waren. Diese Beobachtung ließ ihn die Frage nach Eigenschaften und Ressourcen dieser Menschen stellen, und er entwickelte in Folge sein Konzept der Salutogenese, der Theorie der Entstehung von Gesundheit. Ähnliche Erfahrungen haben Viktor Frankl zu seiner Logotherapie, der dritten Wiener Psychotherapieschule, angeregt.

Das zunehmende Verständnis der Zusammenhänge von Stress und der Reaktion des Organismus auf diesen führte zu der Beobachtung des „Allgemeinen Adaptationssyndroms“ durch Hans Selye (1974), das besagt, dass die Stressantwort des Körpers sich nicht nach der Art des Stressors richtet, sondern nach der wahrgenommenen Notwendigkeit einer Anpassungsreaktion.

Der englische Kinderpsychiater Michael Rutter hat in seinen longitudinalen Studien nachgewiesen, dass Resilienz nicht ausschließlich auf angeborene Stärke oder die Akkumulation von Faktoren, Risiko oder Unterstützung zurückzuführen ist, 
sondern auf eine sich amplifizierende Interaktion dieser verschiedenen Faktoren. Insbesondere Rutters Studie (Rutter et al. 2007) über Kinder, die unter schwersten Bedingungen in rumänischen Waisenhäusern aufwuchsen und nach dem Fall des Ceausescu-Regimes von britischen Familien adoptiert worden waren, hat wesentlich dazu beigetragen, die Folgen von Deprivation besser zu verstehen und die Folgen einer gelingenden Adoption und entsprechender Verbesserung der Entwicklung nachzuweisen.

In den 1970er-Jahren beschrieb der Psychiater George L. Engel (1977) das biopsychosoziale Modell, aufbauend auf den Erkenntnissen der Allgemeinen Systemtheorie, und trug wesentlich zur Entwicklung eines sozialen Krankheitsbegriffs bei, der nicht nur biologisch-medizinische Einflüsse berücksichtigt, sondern das Verständnis von Krankheit um die Dimensionen des Psychosozialen erweiterte.

Aus der Sicht der Erwachsenenmedizin wurde von Egle (2004) und Felliti et al. (1996) das Konzept der Adverse Childhood Experiences - der Auswirkungen von frühkindlichen negativen Ereignissen - eingeführt. Sie beobachteten bei erwachsenen Patienten mit internistischen und/oder psychosomatisch-psychiatrischen Erkrankungen eine überzufällige Häufung negativer Kindheitserlebnisse, wie Traumatisierungen, Scheidung, Tod der Eltern und andere. Mit zunehmender Forschungstätigkeit entwickelte sich ein neues, multimodales und dynamischeres Verständnis der Entwicklung von Krankheit und Gesundheit (Rothenberger und Hüther 1997), in dem das Konzept der Resilienz eine wesentliche Rolle spielt.

\section{Resilienzkriterien}

Resilienzkriterien sind die definitorischen Eigenschaften eines Einflussfaktors, die als risikomindernd, respektive resilient, bezeichnet werden. Sie sollten bei risikoerhöhender Gefährdung wirksam sein, z. B. einen Puffereffekt oder einen kompensatorischen Einfluss haben und so die Auswirkungen von Herausforderungen oder negativen Einflüssen dementsprechend moderieren (Holtmann und Schmidt 2004; Laucht et al. 1997). In den verschiedenen Studien zur Resilienz wurde das sogenannte resiliente Kriterium oft wie folgt definiert:

- das Nicht-Erkranken im Falle einer erblich belasteten Situation

- die Nicht-Entwicklung einer bekannten Folge eines Risikofaktors

- Kriterien des Gelingens äußerer Anpassung und/oder

- gelungene Lebensbewältigung

- die Verbesserung des Sozialkapitals oder anderer sozialer Parameter (Verehelichung, Arbeit, gesellschaftliche Integration)

\section{Resilienzfaktoren}

Die Forschung der letzten Jahrzehnte hat verschiedenste Faktoren gefunden, die einerseits mit einem besseren Outcome oder andererseits einem höheren Risiko verbunden sind. Daher werden Schutzfaktoren und Risikofaktoren für Resilienz 
unterschieden. Die zentrale Erhebung und Beschreibung dieser Faktoren geht auf die Arbeiten Emmy Werners (Werner 1989, 2001, 2002, 2004) zurück.

\subsection{Schutzfaktoren}

Das Konzept der Resilienz- oder Schutzfaktoren beinhaltet jene Faktoren, die zu einer positiven Lebensanpassung bzw. Adaptation führen oder eine Bewältigung der Situation nach sich ziehen (Werner 2004). Fergus und Zimmerman (2005) teilten Resilienzfaktoren in zwei Unterkategorien: Ressourcen und Eigenschaften (Assets). In diesem Zusammenhang werden Ressourcen als externe, Eigenschaften als innere Schutzfaktoren definiert.

In Anlehnung an das sozioökologische Modell von Bronfenbrenner (Mash 2018) unterscheidet man Schutzfaktoren in folgenden Bereichen:

1. der Kultur (Makrosystem)

2. der Gesellschaft (Exosystems)

3. der unmittelbaren Umgebung (Mesosystem, z. B. Nachbarschaft, Schule etc.)

4. der Familie (Mikrosystem)

5. des Individuums

Kulturelle Faktoren sind Wertesysteme, Bräuche, Sprachen einer bestimmten Region, eines Landes. Aufbauend auf diesen Werten organisiert sich eine Gesellschaft in unterschiedlichen Formen. Gesellschaftliche Einflussfaktoren sind die Staatsform, in der die Individuen leben oder die unmittelbaren politischen Auswirkungen von Gesetzen. Umweltfaktoren der unmittelbaren Umgebung sind positive Schulerfahrungen und sichere Nachbarschaft. Weiters zählen verlässliche, unterstützende Bezugspersonen bis ins Erwachsenenalter dazu. Familiale Faktoren sind einerseits eine dauerhaft gute Beziehung zu mindestens einer primären Bezugsperson und andererseits das Vorhandensein einer Großfamilie im Sinne kompensatorischer Elternbeziehungen und Entlastung der (eventuell alleinerziehenden) Mutter. Prinzipiell ist ein wenig konflikthaftes, offenes und auf Selbstständigkeit hin orientiertes Erziehungsklima ein wesentlicher Schutzfaktor für Kinder und Jugendliche. Auf der persönlichen Ebene sind eine überdurchschnittliche Intelligenz sowie ein robustes aktives und kontaktfreudiges Temperament als starke Schutzfaktoren anzusehen, wie auch eine sichere Bindung. Soziale Unterstützung - sei es durch Eltern, Freunde, Verwandte oder Nachbarn - ist einer der zentralen Schutzfaktoren. Aus diesem Verständnis heraus wird deutlich, dass die Interaktion mit den primären Bezugspersonen eine zentrale Bedeutung bei der Entstehung von Resilienz hat. Verschiedene AutorInnen setzen heute auch den Begriff der Resilienz mit dem Begriff der sicheren Bindung gleich.

\subsection{Risiko- oder Belastungsfaktoren}

Die Belastungsfaktoren, die die Vulnerabilität des Individuums erhöhen und damit die Resilienz mindern, finden sich ebenfalls auf allen oben angeführten Ebenen. Gesellschaftliche Faktoren sind beispielsweise Konflikte, Krieg oder Naturkatastrophen. Der am meisten vorkommende und weltweit häufigste soziale Belastungsfaktor 
ist Armut, ein niedriger sozioökonomischer Status (Wadsworth et al. 2018) sowie mangelnde soziale Unterstützung. Eine soziale Situation mit zu großer Familie, zu wenig Raum für Wohnen und die Situierung in einem Ghetto (z. B. Flüchtlingslager) zählen ebenfalls zu Belastungsfaktoren aus dem Umfeld. Im Bereich der Faktoren des näheren Umfeldes der Eltern sind Erkrankungen und/oder Belastungen der Eltern durch die Lebensumstände anzuführen. Häufig wechselnde Beziehungen der Eltern, alleinerziehende Eltern und vor allem die Abwesenheit der Väter sind wesentliche Belastungsfaktoren. Väter mit autoritärem Erziehungsstil und/oder Arbeitslosigkeit stellen für die Familie bzw. die Kinder einen Risikofaktor dar. Die Trennung der Eltern oder der Verlust von Elternteilen zählen gleichfalls wie andere familiale Merkmale - chronisch disharmonisches Familienklima, Gewalt, Misshandlung - oder eine zu knappe Geschwisterfolge ebenfalls zu dieser Gruppe von Faktoren.

Teilweise können für bestimmte Erkrankungen oder Zustände sehr spezifische Risiko- oder Schutzfaktoren gefunden werden. So beschreiben Carballo et al. (2019) psychologische Faktoren (Depression, Angst, vorangegangene Selbstmordversuche), stressvolle Life-Events und Persönlichkeitsmerkmale als signifikante Risikofaktoren für Suizidalität bei Jugendlichen. Ähnliche Ergebnisse liegen für verschiedenste Krankheitsbilder vor wie zum Beispiel für traumatische Kopfverletzungen (Holland und Schmidt 2015) oder Autismus (Szatmari 2017).

Neben den individuellen Faktoren wurden auch für Gruppen spezifische Schutzoder Risikofaktoren beschrieben. Das bedeutet, dass bestimmte Gruppen, Ethnien oder Gesellschaftsschichten über gemeinsame und für die Gruppe spezifische Resilienzfaktoren verfügen. Dies gilt z. B. für Religionen und andere Formen starker ideologischer Gemeinschaft. (z. B. Chamlee-Wright und Zhou 2009) sowie für andere eher organisationsbedingte Gruppen, wie Kindertagestätten (vgl. zum Thema „Resilienzförderung in Kindertageseinrichtung und Grundschule“ auch den Artikel von Fröhlich-Gildhoff und Rönnau-Böse in diesem Heft). Auch für Schulen wurden gruppenresiliente Phänomene beschrieben (Agasisti et al. 2018).

Für ältere Menschen wurden von Madsen et al. (2019) folgende persönliche Faktoren der Resilienz beschrieben: positives Umdeuten, Handlungsbereitschaft, eine eigene Meinung, zweckgerichtetes Handeln, Akzeptanz und Zugehörigkeit. Auf kommunaler Ebene waren Strategien der Selbstbemächtigung und gemeinsame Entscheidungsfindung wie kollektive Handlungsbereitschaft und Engagement resilienzfördernd für ältere MitbürgerInnen.

\section{Modelle der Resilienz}

Angesichts der angeführten zahlreichen Faktoren und Einflüsse drängt sich die Frage auf, wie man sich das Zusammenwirken dieser vorstellen kann. In früheren Modellen wurden Resilienzfaktoren als kompensatorische Faktoren oder als herausfordernde bzw. ,stählende“ Faktoren verstanden. Andere verwendeten das Faktorenmodell im Sinne einer Waage zwischen Schutz- und Risikofaktoren, die auf das Individuum einwirken können. Beide Gruppen von Faktoren können additiv wirken. Für Risikofaktoren gilt: Je mehr vorhanden sind, desto signifikanter nimmt die Resilienz 
des Individuums ab (Jaffee et al. 2007). Für Schutzfaktoren wiederum gilt: Je mehr vorhanden sind, umso größer ist die Resilienz des Individuums.

Mit den Fortschritten der Genetik und der Genforschung wurden auch genetische Einflüsse auf die Resilienz untersucht. Es zeigte sich, dass das Vorliegen eines Allels (Genvarianten) beispielsweise für Depression oder antisoziales Verhalten, nur dann Auswirkungen hat, wenn zusätzlich ein Risikofaktor vorhanden ist (Caspi et al. 2003). Daraus wurde geschlossen, dass der genetische Einfluss durch Interaktion mit Umgebungsfaktoren wirksam wird. Die epigenetische Forschung der letzten Jahre konnte auch Interaktionen zwischen Verhalten und Veränderungen der DNA nachweisen, die in Folge auch generationenübergreifend weitergegeben werden können.

Resilienzfaktoren können daher statisch oder dynamisch sein, d.h. einmalig vorhanden sein, andauernd vorhanden sein oder wechselnd in Ausprägung und Dauer. Resilienz wird demnach heute als ein Prozess definiert, der sowohl von vorbestehenden oder sich entwickelnden Resilienzfaktoren als auch von Anpassungsvorgängen getragen wird. Aus diesem Gesichtspunkt heraus stellt sich die Frage, wie der Mensch Resilienz erwirbt.

Die psychologische Entwicklung des Menschen wird als ein dynamischer Prozess des Individuums auf genetischer, neurobiologischer und psychologischer Ebene gesehen, der mit der direkten und indirekten Umwelt in Interaktion tritt. Durch diese Interaktion verändert sich sowohl das Individuum auf allen Ebenen (neurobiologisch, epigenetisch, sozial) wie auch das umgebende System. In einer von McCrory et al. (2017) publizierten Studie wurden die Ergebnisse der epigenetischen, psychiatrischpsychologischen Forschung bezüglich der posttraumatischen Belastungsstörung und der Reaktion auf Traumatisierungen modellhaft dargestellt. Dieses Modell beinhaltet die Auswirkungen der frühkindlichen negativen Ereignisse - der Adverse Childhood Experiences - im Sinne eines negativen Umgebungsmilieus und ist als ein ineinandergreifender, sich wiederholender Prozess der Persönlichkeitsentwicklung zu verstehen (Strunk und Schiepeck 2008). In diesem Modell wird davon ausgegangen, dass ein Kind, das in einer negativen Umgebung aufwächst, diese Gegebenheiten durch neurokognitive Anpassungsprozesse in sich aufnimmt und verankert. Das hat eine veränderte emotionale Reaktion auf Bedrohung zur Folge, es kommt zu negativen Copingstrategien (Bewältigungsstrategien) etc. und erzeugt so eine latente Vulnerabilität. Durch wiederholte positive/negative Erfahrungen werden diese Systeme immer weiter differenziert, internalisiert und angepasst. Auf der biologischen Ebene führt dies zu Veränderungen der neuro-hormonalen Reaktion, dem Aufbau vulnerabler oder resilienter Netzwerke und Veränderungen der Hirn-Organe (z.B. in der Amygdala oder im präfrontalen Cortex, Bolsinger et al. 2018) und veränderten Handlungsoptionen. Durch diese laufenden Anpassungsvorgänge gewährleistet der Organismus eine Anpassung an die Umgebungsbedingungen. Diese Anpassung erzeugt im negativen Milieu eine nicht-optimale emotionale Regulation, eine veränderte Stressantwort und führt dadurch zu einer erhöhten Vulnerabilität. Und so läuft das Individuum bei neuerlicher Stressbelastung in Gefahr, wieder erneut traumatisiert zu werden. Diese wiederholten Reaktionen führen in Folge zu einem wesentlich erhöhten Risiko z.B. psychisch zu erkranken.

An jeder Stelle dieses Modells besteht die Möglichkeit der Veränderung, der Anpassung des Systems durch Veränderung der äußeren Faktoren oder der Verbesserung 
von Copingstrategien oder Hebung der Resilienzfaktoren. Es handelt sich somit um ein nicht-lineares, dynamisches Modell (Strunk and Schiepeck 2008). Nicht-lineare Modelle haben aber weitreichende Konsequenzen auf unser Verständnis von Gesundheit und Krankheit. Die systemische Sicht ,versteht Gesundheit und Krankheit als kohärente, dynamische Strukturen, die beide Ausdruck des biopsychosozialen Systems sind" (ebd.). Gesundheit und Krankheit sind also in unserem menschlichen System stets gleichzeitig vorhanden und die gesunden Anteile jederzeit nützbar, z. B. bei Mobilisierung der subjektiven Heilungskräfte. Somit ist auch klar, dass es aus diesem Verständnis heraus - zumindest für psychische Erkrankungen - keine direkte Ursache-Wirkungsbeziehung gibt. Die Auswirkungen von Interventionen (Schicksalsschlägen, Traumata, Behandlungen etc.) sind folglich in so einem System auch nicht direkt vorhersehbar und prognostizierbar.

Die neurobiologische Forschung bestätigt diese Modelle insofern, als heute klar ist, dass das Gehirn sich zeitlebens verändern kann (Plastizität), dass die wiederholte Anwendung bestimmter Reaktionen zu immer besser vernetzten Strukturen (,Netzwerkkarten“) führt, was eine zunehmende Verbesserung der Antwort und gleichzeitig ein Einsparen von Ressourcen ermöglicht (Bolsinger et al. 2018). Dies gilt natürlich für resiliente und nicht-resiliente Aktionen gleichermaßen. Insbesondere frühe Einflüsse (Adverse Childhood Experiences) führen zu Veränderungen von Hirnregionen mit Auswirkungen auf die Emotionsverarbeitung und Selbststeuerung (Bolsinger et al. 2018; Callaghan et al. 2013) und auf die Entstehung psychischer Erkrankungen.

\section{Definition von Resilienz}

Resilienz ist keine ausschließlich angeborene Fähigkeit, aber auch nicht ausschließlich das Resultat negativer oder positiver (Umwelt-) Einflüsse. Resilienz entwickelt sich über die Zeit. Somit erklärt sich Resilienz als ein allgemein menschliches Phänomen, das aus dem Zusammenwirken basaler humaner, adaptiver Systeme mit der Umwelt entsteht, um den Menschen zu befähigen, schwierige Lebenssituation zu bewältigen. Sind diese basalen Mechanismen geschützt und gepflegt, kann sich der Mensch robust entwickeln, selbst unter der Einwirkung negativer Faktoren (Stainton et al. 2018). Etwas kürzer gefasst ist Resilienz (Southwick et al. 2014a) das gesunde, adaptive und/oder integrative Funktionieren über die Zeit nach einem traumatisierenden Erlebnis (zur Abgrenzung von Resilienz zum Phänomen des posttraumatischen Wachstums vgl. den Beitrag von Mangelsdorf in diesem Heft). In Abb. 1 findet sich der Versuch, den Resilienzprozess anhand des bisher Berichteten übersichtlich darzustellen.

Soweit eine allgemeine Definition. In der Forschung wird aufgrund der weitläufigen Verwendung des Begriffes zunehmend eine Spezifizierung des Begriffes gefordert (Rönnau-Böse und Fröhlich-Gildhoff 2011; Southwick et al. 2014b). Jeweils zu definieren ist, ob von Resilienz im Sinne einer stabilen Reaktionsfähigkeit auf akute Ereignisse gesprochen wird (verbunden mit einer vorübergehenden Dysbalance, stable resilience trajectory) oder von Resilienzprozessen, die angesichts chronischer Belastungen (Armut, Krieg) gelingen (emergent resilience). Von 


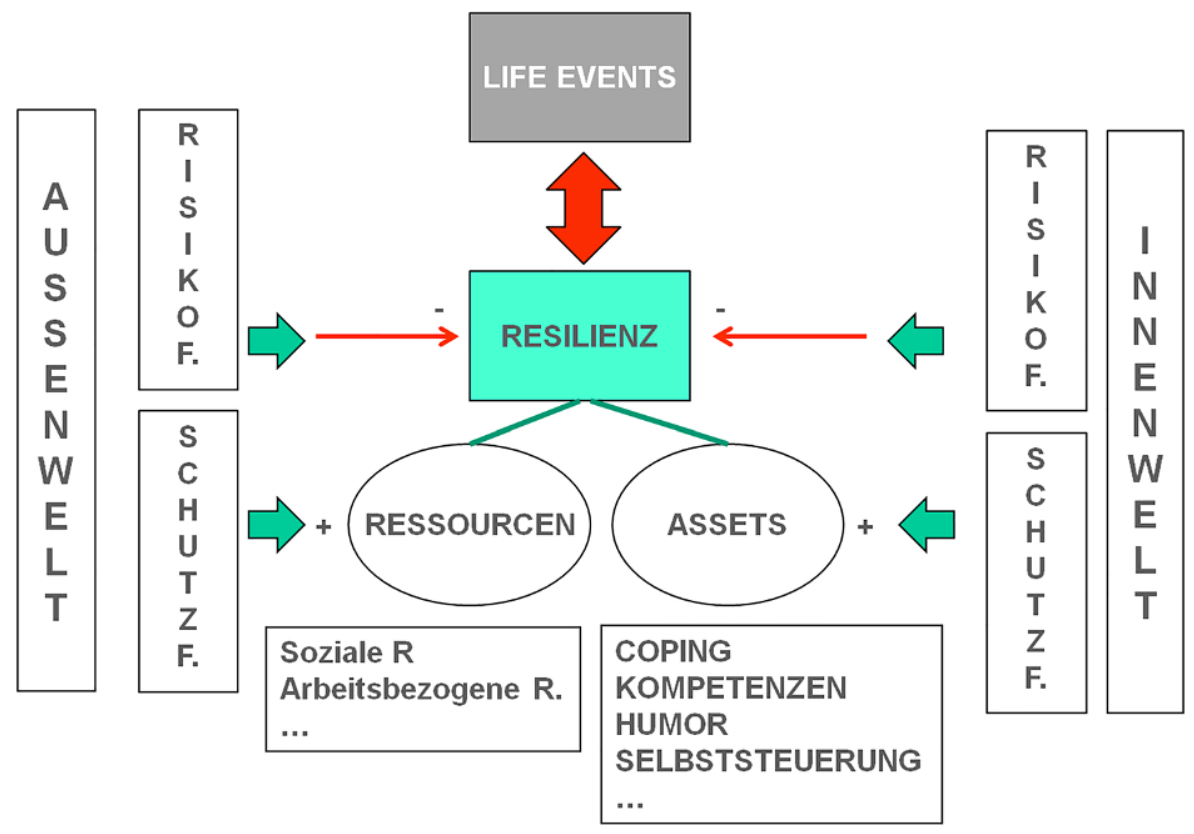

Abb. 1 Zusammenschau der Befunde zum Prozess der Resilienz

„minimal impact resilience“ wird gesprochen, wenn es um Reaktionen auf akute Stressfaktoren/Traumata oder potentiell traumatisierende Ereignisse geht.

\section{Bedeutung des Resilienzmodells für die Behandlung}

Das Verständnis der Resilienz als entwicklungspsychologischer Prozess über die gesamte Lebenszeit ist grundlegend für die Arbeit mit KlientInnen oder PatientInnen, Organisationen oder Gruppen und kann, ja soll das reale Handeln in der Therapie beeinflussen (Ungar 2011). Dieses Verständnis der Resilienz verlangt nach einem gründlichen Kennenlernen der Lebens- respektive Entwicklungsgeschichte mit ihren Umgebungsbedingungen und Einflussfaktoren. Das Besondere in der Therapie ist, dass der/die KlientIn selbst beurteilt, was für ihn/sie hilfreiche oder belastende Einflüsse waren. Gemeinsam ist es dann möglich, dieses Narrativ im Spiegel des heutigen Wissens zu reflektieren und so ein gemeinsames Verständnis der Geschichte des/der KlientIn zu erreichen und modellhaft darzustellen. Bei der Anwendung des Resilienzkonzeptes hilft das salutogenetische Konzept von Anton Antonovsky (1997). Das salutogenetische Dreieck (Abb. 2) hat viele der in der heutigen Traumatherapie selbstverständlichen Positionen vorweggenommen. Zentrales Thema der Gesundung ist das Kohärenzgefühl, das sich aus drei Teilen zusammensetzt: der Verstehbarkeit (die Fähigkeit, die Zusammenhänge des Lebens zu verstehen), der Handhabbarkeit (die Überzeugung, dass man das eigene Leben gestalten kann) und der Sinnhaftigkeit (dem Glauben, dass das Leben Sinn hat). Verschiedene 


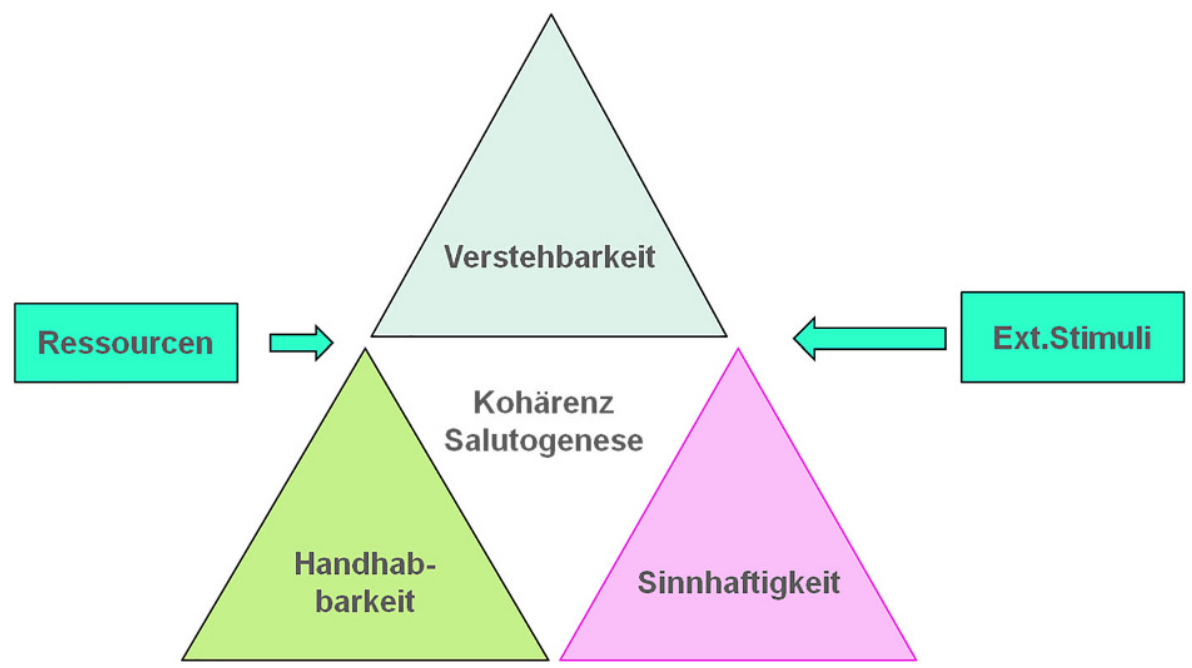

Abb. 2 Dreieck der Salutogenese nach Anton Antonovsky (1997)

AutorInnen haben diese Ansätze genützt, um Resilienz und deren Förderung handhabbar zu machen. Es wurden sieben Einflussfaktoren auf die Resilienzentwicklung beschrieben (Deinert 2015), die einer Bearbeitung in der Therapie zum Teil gut zugänglich erscheinen: Optimismus, Akzeptanz, Lösungsorientierung, Opferrolle verlassen, Verantwortung übernehmen, Netzwerkorientierung und Zukunftsplanung. Laut Rönnau-Böse und Fröhlich-Gildhoff (2011) decken sich diese Faktoren mit den von der WHO bereits 1994 definierten „Lebenskompetenzen“. In der Umsetzung müssen diese Skills mit und für den/die KlientIn/PatientIn praktisch handhabbar und messbar gemacht werden (ebd.).

Das Resilienzkonzept ermöglicht, sich aus der reinen Belastungs- und Stressorientierung in eine ökologische und sozialorientierte Betrachtungsweise der Lebenssituation zu bewegen und damit ein weites Feld für Interventionen zu öffnen (Ungar 2011). Resilienzförderung muss neben der notwendigen Individuumszentrierung immer auch das soziale Umfeld mitdenken und einplanen, also auch Interventionen in diesen Bereichen anstoßen (Familie, Kinder- und Jugendhilfe, Soziale Hilfen etc.). Es erfordert weiters die Erforschung von Prozessen, denen persönliche Stärke zugrunde liegt (Ressourcen- und Lösungsorientierung). Weitere persönliche Systeme, deren Integration die Resilienzorientierung erleichtert, sind die Nutzung des Bindungssystems, des Selbststeuerungssystems und des Bewältigungsmotivations-Systems (Kleinhanns 2008). Hilfreich ist dabei auch, dass der Resilienzprozess kein linearer Prozess ist und viele Faktoren diesen Prozess zu verschiedenen Zeitpunkten und mit unterschiedlicher Intensität und Bedeutung beeinflussen. Der Grundsatz, dass viele Wege zum Ziel führen können, wird hiermit untermauert und ermöglicht ein vielfältiges therapeutisches Herangehen.

Die dauerhafte und verlässliche Beziehung zu einer Bezugsperson ist einer der zentralen Resilienzfaktoren und so ist auch die Beziehung zum/zur TherapeutIn ein wesentlicher Resilienzfaktor und dadurch besonders kostbar und bedeutsam. 
Diese Beziehung ermöglicht im Idealfall Wachstumsprozesse, die Bereitschaft zum Handeln, die Fähigkeit zu reflektieren und unterstützt die Fähigkeit zur Neubewertung und positiven Umdeutung einer Situation. Dabei ist ein hohes Maß an ,,sensibler Kontextbezogenheit" nötig, ein sensibles, wissendes Erkennen der vielschichtigen Zusammenhänge im Moment der Therapie. Die eigene Resilienzentwicklung im Umgang mit dem/der KlientIn/PatientIn in der Therapie ist nach Buchholz (2012) Grundlage dieser ,,sensiblen Kontextbezogenheit“ in der professionellen Rolle des/der TherapeutIn. Erst wenn diese Erfahrungen für die Therapie nutzbar sind, entsteht ein tieferes Verständnis des Gegenübers. Dabei ist auch zu berücksichtigen, dass Resilienz ohne Vulnerabilität nicht denkbar ist. Bei Verlust des einen oder anderen Pols sind krankhafte Prozesse im Spiel. Das bedeutet, um resilient werden zu können, muss Vulnerabilität zugelassen werden und der Mensch sich seinen Schwächen stellen können (ebd.).

\section{Psychodrama und Resilienz}

Jacob Levy Moreno (1889-1974) hat in seinen Grundideen den Begriff der Spontaneität in seine Modelle eingeführt. Sein Verständnis der Spontaneität ist, dass ein System, egal ob Individuum oder Gesellschaft, auf eine neue Situation und auf eine bekannte sich wiederholende Situation angemessen reagieren kann. Sein Prozessmodell - der kreative Zirkel - (Bender und Stadler 2012) beschreibt die Entwicklung anhand einer Herausforderung unter der Beteiligung von Spontaneität, Kreativität und Rolle. Dieses Kreismodell kann als ein frühes Resilienzmodell angesehen werden, in dem sowohl die externen Faktoren einen Platz finden als auch Herausforderungen und Veränderungsansprüche, aber auch der innere Zustand (Spontaneität, Kreativität und Veränderungsbereitschaft). Gleichzeitig ist dieses Modell auch Morenos Störungsmodell. Salutogenetische, aber auch pathogenetische Faktoren können an jeder beliebigen Stelle dieses Zyklus' auftauchen und den kreativen Prozess fördern, behindern oder gefährden. Dieses ursprüngliche Modell von Moreno wurde von Schacht und Krüger erweitert und dem modernen Wissensstand angepasst. In Schachts Modell erfordert eine gegebene Ausgangslage entweder ein Verhalten, das der Mensch bereits in seinem Repertoire hat oder es neu erzeugen/erlernen muss (Schacht 2009). Das Gelingen dieses adäquaten, angepassten Verhaltens hängt von verschiedenen Bedingungen ab, die mit den Erfahrungen während der Entwicklung der Persönlichkeit zu tun haben. Es steht in direktem Zusammenhang mit Ressourcen, Copingstrategien und den positiven Erfahrungen bzw. mit den Vulnerabilitätsfaktoren und persönlichen Faktoren, wie dem Strukturniveau, der Motivation und andere Faktoren. In Krügers Modell geht es um die störungsorientierte Sichtweise der Psychodramatechniken (Krüger 2002). Nach Krüger würden zu jedem Krankheitsbild spezifische Blockaden (Risiken) des kreativen Selbstorganisationsprozesses vorhanden sein, die sich auf unterschiedlichen Funktionsniveaus jeweils anders auswirken (Systemorganisation, Realitätsorganisation, Kausalitätsorganisation etc.). Durch das Zur-Verfügung-Stellen adaptiver und kreativer Rollenmodelle, der regulatorischen Anpassung von Gefühlen und Gedanken wird eine Weiterentwicklung, respektive die Entwicklung kreativen und adäquaten Verhaltens unterstützt. 
Das Psychodrama ist also von Anfang an als resilienzfördernde Methode angelegt. Moreno selbst hat beispielsweise als Indikation für seine soziometrische Arbeit das Ziel der Förderung von sozialer Kompetenz und Stärke genannt (Aichinger 2010). Verschiedene AutorInnen haben sich mit der resilienzfördernden Wirkung des Psychodramas auseinandergesetzt und seine positive Wirkung auf die Resilienzentwicklung aufzeigen können. Als wirksame Methoden sind zum Beispiel die Märchenarbeit (Walters 2017), der Zauberladen (Deinert 2015) oder die spezifische Herangehensweise (Teilearbeit) von Alfons Aichinger (Aichinger 2010; Aichinger und Holl 1997) zu nennen. Rainer Bosselmann (1996) beschreibt das Spiel auf der leeren Bühne und die damit verbundene Herstellung einer Surplus Reality als zentrale Methode. Die Inszenierung auf der leeren Bühne ermöglicht, die eigene Lebenssituation mit szenischen Mitteln unter eigener Regie körperlich handelnd erfahrbar zu machen. In der abschließenden Integrationsphase wird mittels Mentalisierungstechniken (Rollenfeedback, Sharing) die Spielerfahrung kognitiv reflektiert, neu bewertet und integriert.

\section{Kritik am Resilienzmodell respektive an seiner Anwendung}

Das zunehmende Verständnis dieser Zusammenhänge wie auch die nachhaltige und intensive Forschung zu Traumafolgen und deren Behandlung haben nach Ottomeyer und Reddemann (2017) sowie Gebauer (2017) zu einer deutlichen Verschiebung der Interpretation des Begriffes der Resilienz geführt. Nach Ottomeyer und Reddemann geht es verschiedenen AutorInnen zunehmend darum, die Umstände als gegeben zu nehmen und die Resilienzentwicklung dem Individuum oder seiner Familie zu überlassen. Im Sinne dieses - eher neoliberalen - Denkansatzes verschiebt sich die sozialpolitische Verantwortung hin zum Individuum und individualisiert gesellschaftliche Risiken. Ein so individualisiertes Verständnis der Resilienz greift zu kurz. Wenn unsere dynamischen Überlegungen zur Resilienz stimmen, ist diese individuumszentrierte Sicht auch fachlich falsch. Bestimmte Situationen wie Kriege, klimabedingte Katastrophen, Migrationsströme oder wirtschaftliche Dauerkrisen sind selbst für höchst resiliente Personen nicht veränderbar. Gesellschaft und Politik können nicht aus der Verantwortung entlassen werden.

Funding Open access funding provided by Paracelsus Medical University.

Open Access Dieser Artikel wird unter der Creative Commons Namensnennung 4.0 International Lizenz veröffentlicht, welche die Nutzung, Vervielfältigung, Bearbeitung, Verbreitung und Wiedergabe in jeglichem Medium und Format erlaubt, sofern Sie den/die ursprünglichen Autor(en) und die Quelle ordnungsgemäß nennen, einen Link zur Creative Commons Lizenz beifügen und angeben, ob Änderungen vorgenommen wurden.

Die in diesem Artikel enthaltenen Bilder und sonstiges Drittmaterial unterliegen ebenfalls der genannten Creative Commons Lizenz, sofern sich aus der Abbildungslegende nichts anderes ergibt. Sofern das betreffende Material nicht unter der genannten Creative Commons Lizenz steht und die betreffende Handlung nicht nach gesetzlichen Vorschriften erlaubt ist, ist für die oben aufgeführten Weiterverwendungen des Materials die Einwilligung des jeweiligen Rechteinhabers einzuholen.

Weitere Details zur Lizenz entnehmen Sie bitte der Lizenzinformation auf http://creativecommons.org/ licenses/by/4.0/deed.de. 


\section{Literatur}

Agasisti, T., Avvasati, F., Borgovoni, F., \& Langobardi, S. (2018). Academic resilience: what schools and countries do to help disadvantaged students in PISA. In A. Schleicher (Hrsg.), OECD Education Working Papers No. 167. Paris: OECD.

Aichinger, A. (2010). Resilienzförderung mit Kindern. Wiesbaden: VS Springer.

Aichinger, A., \& Holl, W. (1997). Psychodrama-Gruppentherapie mit Kindern. Mainz: Mathias Grünewald Verlag.

Antonovsky, A. (1997). Salutogenese. Zur Entmystifizierung der Gesundheit. Tübingen: dgvt.

Bender, W., \& Stadler, C. (2012). Psychodrama-Therapie: Grundlagen, Methodik und Anwendungen. Stuttgart: Schattauer.

Block, J. (1977). The developmental continuity of EGO-control and EGO-resiliency: some accomplishements. Conference paper. Society for Research in Child Development, New Orleans..

Bolsinger, J., Seifritz, E., Kleim, B., \& Manoliu, A. (2018). Neuroimaging correlates of resilience to traumatic events-A comprehensive review. Frontiers in Psychiatry. https://doi.org/10.3389/fpsyt.2018. 00693.

Bosselmann, R. (1996). Das Psychodrama und seine Heilfaktoren. Meezen: Limmer Verlag.

Buchholz, M.B. (2012). Resilienz - die Entwicklung professioneller Therapeuten und die Bewältigung therapeutischer Paradoxien. In W. Weigand (Hrsg.), Philosophie und Handwerk der Supervision (S. 159-187). Gießen: Psychosozial Verlag.

Callaghan, B.L., Graham, B.M., \& Richardson, R. (2013). From resilience to vulnerability: mechanistic insights into the effects of stress on transitions in critical period plasticity. Frontiers in Psychiatry, 4 , 90.

Caspi, A., Sugden, K., Moffit, T. E., Taylor, A., Craig, I. W., Harrington, H., \& Poulton, R. (2003). Influence of life stress on depression: moderation by a polymorphism in the 5-HTT gene. Science, 301, 4.

Chamlee-Wright, E., \& Zhou, M. (2009). Club Goods and post-disaster community return. Rationality and Society, 21, 4.

Deinert, K. (2015). Morenos Stehaufmännchen. Zusammenhang zwischen Psychodrama und Resilienzentwicklung. Diplomarbeit. Hamburg: Institut für soziale Interaktion (ISI).

Egle, U.T. (2004). Long-term effects of adverse childhood experiences-Actual evidence and needs for research. Z Psychosom Med Pychother, 48(4), 411-434.

Engel, G.L. (1977). The need for a new medical model: a challenge for biomedicine. Science, 196(4286), 129-136.

Felitti, V. J., Anda, R. F., Nordenberg, D., Williamson, D. F., Edwards, V., Koss, M. P., \& Marks, S. J. (1996). Relationship of childhood abuse and household dysfunction to many of the leading causes of death in adults. Am J Prev Med, 14(4), 245-258.

Fergus, S., \& Zimmerman, M. (2005). Adolescent resilience: a framwork for understanding healthy development in the face of risk. Ann Rev Publich Health, 26, 399-419.

Garmezy, N. (1991). Resilience in children's adaptation to negative life events and stressed environments. Pediatrics, 20, 459-466.

Garmezy, N., \& Masten, A.S. (1984). The study of stress and competence in children: a building block for developmental psychopathology. Child Development, 55, 97-111.

Gebauer, T. (2017). Fit für die Katastrophe. In medico international (Hrsg.), Fit für die Katastrophe? (S. 13-22). Gießen: Psychosozial Verlag.

Holland, J. N., \& Schmidt, A.T. (2015). Static and dynamic factors promoting resilience following brain injury: a brief review. Neural Plasticity. https://doi.org/10.1155/2015/902802.

Holtmann, M., \& Schmidt, M. H. (2004). Resilienz im Kindes- und Jugendalter. Kindheit und Entwicklung, 13(4), 195-200.

Jaffee, S. R., Caspi, A., Moffit, T.E., Polo-Tomas, M., \& Taylor, A. (2007). Individual, family and neighborhood factors distinguish resilient from non-resilient maltreated children: a cumulative stressors model. Child Abuse \& Neglect, 31, 231-253.

Kleinhanns, J. (2008). Resilienzförderung im Kinderpsychodrama. Zeitschrift für Psychodrama und Soziometrie, 2/2008, 225-242.

Krüger, R. T. (2002). Wie wirkt Psychodrama? Der kreative Prozess als übergeordnetes Theoriekonzept des Psychodramas. Zeitschrift für Psychodrama und Soziometrie, 2/2002, 273-316.

Laucht, M., Esser, G., \& Schmidt, M.H. (1997). Wovor schützen Schutzfaktoren? Anmerkungen zu einem populären Konzept der modernen Gesundheitsforschung. Zeitschrift für Entwicklungspsychologie und Pädagogische Psychologie, 29, 260-270. 
Madsen, W., Ambrens, M., \& Ohl, M. (2019). Enhancing resilience in community-dwelling older adults: a rapid review of the evidence and implications for public health. Front Public Health, 7, 14.

Mash, E. J. (2018). Abnormal child psychology. Boston: Mindtap Course List.

McCrory, E. J., Gerin, M. I., \& Viding, E. (2017). Annual research review: childhood maltreatment, latrent vulnerability and the shift to preventative psychiatry - the contribution of functional brain imaging. J Child Psychol Psychiatr, 58(4), 338-357.

Ottomeyer, K., \& Reddemann, L. (2017). Die Suche nach dem guten Leben. In medico international (Hrsg.), Fit für die Katastrophe? (S. 35-57). Gießen: Psychosozial Verlag.

Rönnau-Böse, M., \& Fröhlich-Gildhoff, K. (2011). Präventionsprogramm für Kindertageseinrichtungen - Förderung von seelischer Gesundheit und Resilienz. In K. Fröhlich-Gildhoff (Hrsg.), Handbuch Resilienzförderung. Wiebaden: VS Springer.

Rothenberger, A., \& Hüther, G. (1997). Die Bedeutung von psychosozialem Streß im Kindesalter für die strukturelle und funktionelle Hirnreifung: neurobiologische Grundlagen der Entwicklungspsychopathologie. Prax Kinderpsychol Psychiatr, 46(9), 623-644.

Rutter, M., Colvert, E., Kreppner, J., Beckett, C., Castle, J., \& Groothues, C. (2007). Early adolescent outcomes for institutionally-deprived and non-deprived adoptees. I: disinhibited attachment. J Child Psychol Psychiatr, 48, 17-30.

Schacht, M. (2009). Das Ziel ist im Weg - Störungsverständnis und Therapieprozess im Psychodrama. Wiesbaden: VS.

Selye, H. (1974). Stress: Bewältigung und Lebensgewinn. München: Piper Verlag.

Southwick, S., Sippel, L., Krystal, J., Charney, D., Mayes, L., \& Pietzrak, R. H. (2014a). Why are some individuals more resilient than others: the role of social support. World Psychiatry, 15(1), 3.

Southwick, S. M., Bonanno, G. A., Masten, A. S., \& Yehuda, R. (2014b). Resilience definitions, theory and challenges: intedisciplinary perspective. Eur J Psychotraumatology. https://doi.org/10.3402/ejpt.v5. 25338. eCollection 2014.

Stainton, A., Chisholm, K., Kaiser, N., Rosen, M., Upthegrove, R., Ruhrmann, S., \& Wood, S. J. (2018). Resilience as a multimodal dynamic process. Early Intervention in Psychiatry, 13, 725-732.

STOP Consortium, Carballo, J.J., Lorente, C., Kehrmann, L., et al. (2019). Psychosocial risk factors for suicidality in children und adolescents. Eur Child Adolesc Psychiatry. https://doi.org/10.1007/ s00787-018-01270-9.

Strunk, G., \& Schiepeck, G. (2008). Systemische Psychologie. München: Spektrum Akadmischer Verlag.

Szatmari, P. (2017). Risk and resilience in autism spectrum disorder: a missed translational opportunity. Dev Med Child Neurol, 60, 225-229.

Ungar, M. (2011). Theorie in die Tat umsetzen. Fünf Prinzipien der Intervention, Handbuch Resilienzförderung (S. 157-187). Wiesbaden: VS Springer.

Wadsworth, M. E., Ahlkvist, J. A., McDonald, A., \& Tilghman-Osborne, E. M. (2018). Future directions in research and intervention with youth poverty. J Clin Child Adolesc Psychol, 47(6), 1023-1038.

Walters, R. (2017). Fairy tales, psychodrama and action methods: ways of helping traumatized children to heal. Z Psychodrama Soziom, 16, 53-60.

Werner, E. E. (1989). A longitudinal study from birth to 32 years. Am J Orthopsychiatry, 59(1), 72-81.

Werner, E.E. (2001). Journeys from childhood to midlife: risk, resilience and recovery. Ithaka: Cornell University Press.

Werner, E.E. (2002). Looking for trouble in paradise: some lessons learned from the Kauai Longitudinal Study. In E. Phelps, F. F. Furstenberg \& A. Colby (Hrsg.), Looking at lives: American longitudinal studies in the twentieth century (S. 297-314). New York: SAGE.

Werner, E. E. (2004). Journeys from childhood to midlife: risk, resilience and recovery. Pediatrics, 114, 492. 

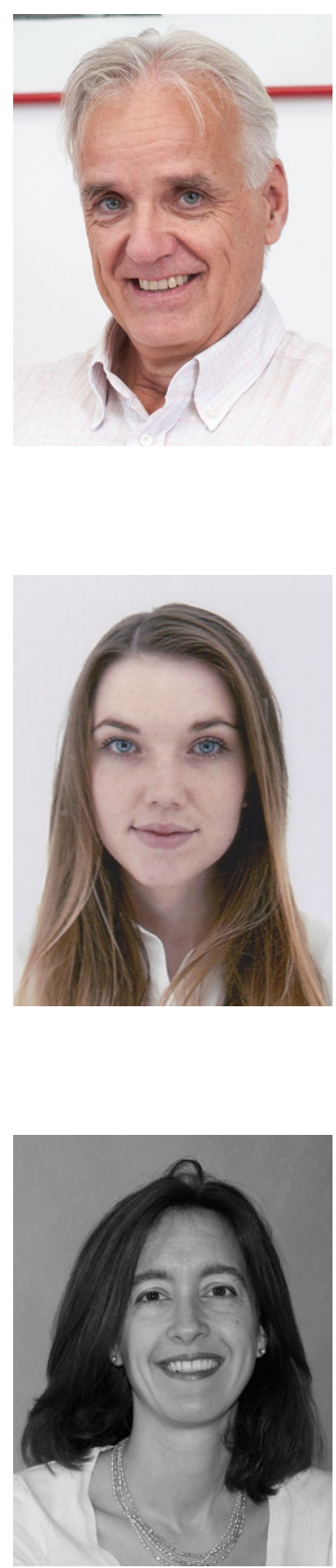

Prim. Univ. Prof. Dr. Leonhard Thun-Hohenstein 1955, Dr. med., Prim. Univ. Prof. Dr., Facharzt für Kinder- und Jugendpsychiatrie und psychotherapeutische Medizin, Zusatzfach Neuropädiatrie, Psychodramatherapeut, Vorstand der Universitätsklinik für Kinder- und Jugendpsychiatrie, Universitätsklinikum Salzburg, Campus CDK, Paracelsus Medizinische Privatuniversität. Lehrauftrag „Soziale Kompetenz im Humanmedizinstudium“ seit 2009. Präsident der Österreichischen Gesellschaft für Kinder- und Jugendpsychiatrie (ÖGKJP, 2018-2020), Aufsichtsrat ProMente Salzburg, Präsidiumsmitglied der Österreichischen Gesellschaft für Kinderschutz in der Medizin (ÖGKiM).

Kerstin Lampert 1992, BA, Bachelorstudium in USA und Thailand, Medizinstudium an der Paracelsus Medizinische Privatuniversität seit 2017. Wissenschaftliche Mitarbeiterin der Universitätsklinik für Kinder- und Jugendpsychiatrie, Universitätsklinikum Salzburg, seit 03/18.

Ulrike Altendorfer-Kling 1972, Dr. med., Fachärztin für Kinderund Jugendpsychiatrie und psychotherapeutische Medizin, Ärztliche Leiterin der Kinder-Jugend-Seelenhilfe Salzburg, Lehrtherapeutin mit partieller Lehrbefugnis für Psychodrama, Soziometrie und Rollenspiel (ÖAGG). Mitglied der ÖGKJP, Lehrende an der Paracelsus Medizinischen Privatuniversität Salzburg, Referentin für Psychosoziale Medizin und Lehrbeauftragte der Österreichischen Ärztekammer, PsychodramaPsychotherapeutin in freier Praxis mit Weiterbildung in Säuglings-, Kinder- und Jugendlichenpsychotherapie. 